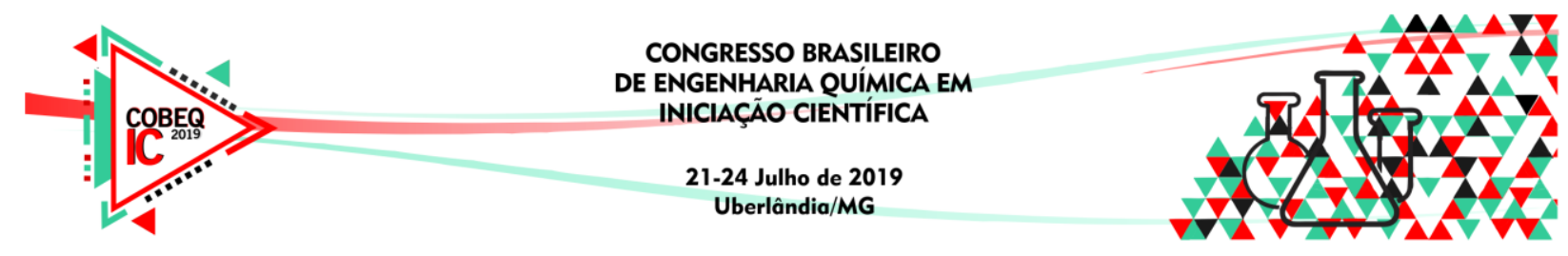

\title{
AVALIAÇÃO DA INFLUÊNCIA DA TEMPERATURA EM UMA COLUNA DE REGENERAÇÃO DE FeIII(EDTA) PARA REMOÇÃO DE H2S EM UMA LINHA DE BIOGÁS
}

\author{
G. L. D. PEREIRA ${ }^{1}$, A. R. IGARASHI, F. N. C. ALMEIDA, D.C. S. MAIA, N. C. PEREIRA \\ Universidade Estadual de Maringá, Departamento de Engenharia Química \\ ra107321@uem.br ${ }^{1}$
}

\begin{abstract}
RESUMO - O sulfeto de hidrogênio pode ser removido de correntes gasosas, como biogás, por meio do processo de absorção oxidativa com soluções aquosas de Fe/EDTA, produzindo o enxofre elementar. A coluna de regeneração pode ser empregada para oxidar o $\mathrm{Fe}$ (II)EDTA para Fe(III)EDTA e reutilizar a solução quelante para repetir o ciclo. Deste modo, objetiva-se observar qual a influência da temperatura de reação na oxidação de Fe(II)EDTA em uma coluna de regeneração. Após a reação com o biogás sintético, variou-se a temperatura de oxidação de $15^{\circ} \mathrm{C}$ à $55^{\circ} \mathrm{C}$ e mantendo constantes a vazão de oxigênio, o pH inicial e a concentração iniciais da reação. Observou-se que a reação ocorre mais rapidamente para temperaturas menores. Como a solubilidade do gás oxigênio aumenta para temperaturas cada vez mais baixas, há uma maior concentração de oxigênio em temperaturas menores, o que aumenta a taxa de oxidação.
\end{abstract}

\section{INTRODUÇÃO}

A produção de biogás é uma valiosa fonte de energia renovável, com potencial para aliviar parcialmente a dependência mundial de combustíveis fósseis, oferecendo uma solução integrada, competitiva e ambientalmente sustentável. Além disso, existem outros aspectos benéficos, como o tratamento de resíduos, produção de biofertilizantes e redução da emissão dos gases do efeito estufa (NADALETTI et al., 2017). O biogás é considerado como fonte renovável de energia, pois é produzido através da digestão anaeróbia, processo biológico que converte a matéria orgânica de resíduos provenientes da agricultura, da pecuária e de indústrias em biogás na ausência de oxigênio (ZHANG, HU e LEE, 2016).

O biogás é composto, principalmente, por 40-75\% de $\mathrm{CH}_{4}, 15-60 \%$ de $\mathrm{CO}_{2}$ e constituintes menores, como $\mathrm{H}_{2} \mathrm{~S}$ e $\mathrm{NH}_{3}$. A elevada concentração de metano torna o biogás um combustível atraente, podendo ser utilizado em uma variedade de aplicações domésticas e industriais, como para geração de energia térmica e elétrica, produção de biometano e como combustível para transporte sustentável (YANG et al., 2014). Embora esteja presente em pequenas quantidades, o sulfeto de hidrogênio é o principal contaminante no uso do biogás para geração de energia elétrica e como combustível. A presença do $\mathrm{H}_{2} \mathrm{~S}$ acelera a corrosão de equipamentos e tubulações, reduzindo seu tempo de vida útil, além de provocar a chuva ácida. Portanto, a dessulfurização é um pré-requisito para o uso do biogás como fonte de energia (TER MAAT, HOGENDOORN e VERSTEEG, 2005). 


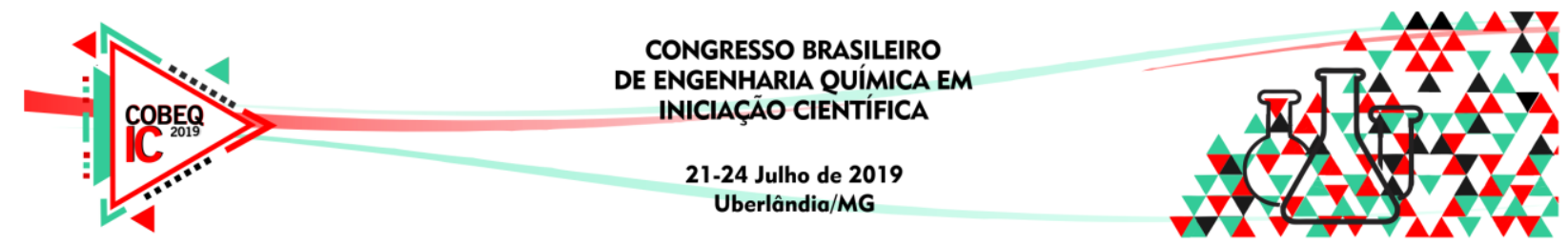

Muitos processos comerciais estão disponíveis para a remoção de sulfeto de hidrogênio a partir de correntes gasosas. A maioria dos processos usa contatores gás-líquidos, nos quais o sulfeto de hidrogênio é colocado em contato com um reagente. Um exemplo de reagente que pode ser empregado é o quelato de ferro (DESHMUKH, G. E SHETE, A., 2012).

Neste processo o sulfeto de hidrogênio é facilmente oxidado a enxofre elementar quando dissolvido em uma solução de Fe/EDTA, simultaneamente, o ferro $\mathrm{Fe}^{3+}$ é reduzido a $\mathrm{Fe}^{2+}$ e se torna inativo. Assim, o composto Fe(II)EDTA é regenerado para a forma férrica pela oxidação da solução com o oxigênio. O processo pode ser descrito pelas Equações 1 e 2 (FRARE et al., 2010):

$$
\begin{aligned}
& \mathrm{H}_{2} S_{(g)} \rightarrow \mathrm{H}_{2} S_{(a q)} \\
& H_{2} S_{(a q)}+2 \mathrm{Fe}^{+3} / \operatorname{EDTA}_{(a q)} \rightarrow S \downarrow+2 H^{+}
\end{aligned}
$$

De acordo com Chuichulcherm et al. (2017), a regeneração de Fe(II)EDTA é um obstáculo à implementação do processo de remoção de $\mathrm{H}_{2} \mathrm{~S}$ em soluções queladas na indústria. A oxidação química espontânea de íons ferrosos a férricos por $\mathrm{O}_{2}$ é um processo complexo, que envolve uma variedade de espécies intermediárias. O intermediário, e consequentemente, o produto final formado depende das condições do meio, tais como $\mathrm{pH}$, temperatura, composição da solução e taxa de oxigênio (Morgan e Lahav, 2007). Por este motivo, o presente trabalho tem como objetivo avaliar a influência da temperatura $\left(15^{\circ} \mathrm{C}\right.$ a $55^{\circ} \mathrm{C}$ ) na regeneração de $\mathrm{Fe}(\mathrm{III}) \mathrm{EDTA}$, em uma coluna de regeneração.

\section{MATERIAIS E MÉTODOS}

\subsection{Módulo Experimental}

Figura 1 - Módulo experimental

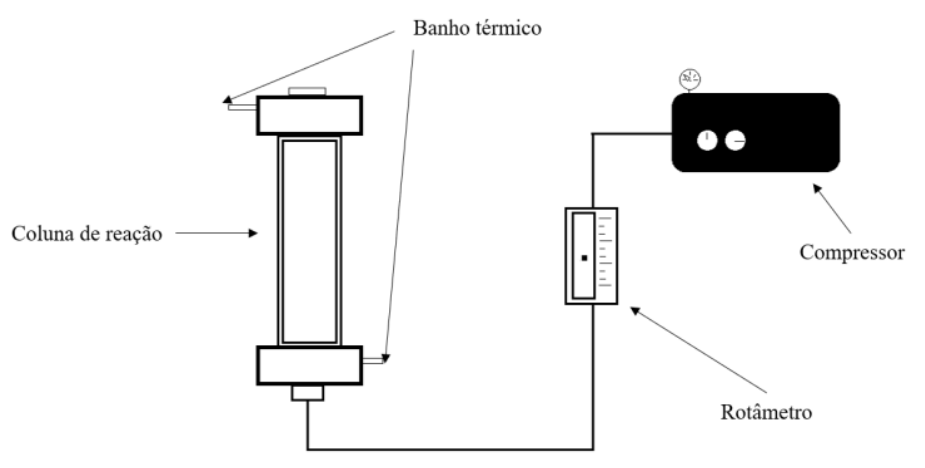

A solução de Fe/EDTA foi preparada com concentração de 0,065 mol/L e pH de 8,98 ajustado com $\mathrm{Na}_{2} \mathrm{CO}_{3}$. Os experimentos foram realizados no módulo experimental (Figura 1) em duas etapas: (1) purificação do biogás, e (2) regeneração da solução.

Inicialmente, na etapa de purificação, a coluna foi preenchida com $0,170 \mathrm{~mL}$ de solução e aguardou-se estabilizar a temperatura, de acordo com experimento. $\mathrm{O}$ biogás 


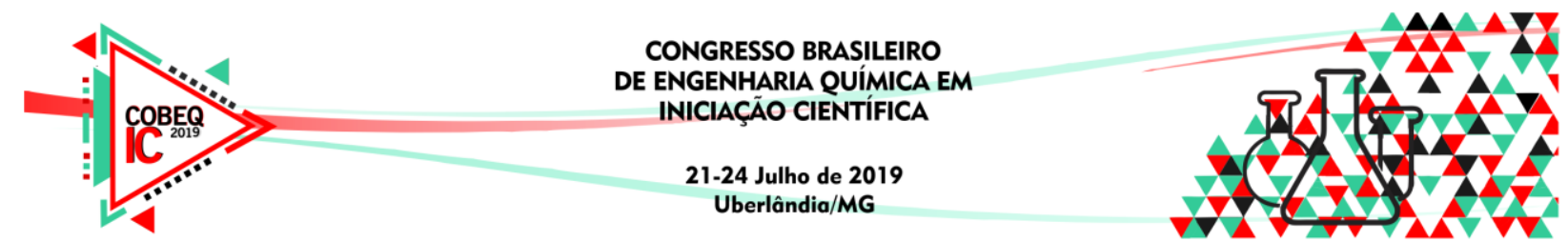

sintético foi injetado na coluna de bolhas com pressão constante de 1 bar e vazão de 80 $\mathrm{mL} / \mathrm{min}$. A reação foi interrompida quando o gás de exaustão ultrapassou a concentração de 10 ppm de H2S, ou seja, quando a solução apresentou a coloração cinza escuro.

Em seguida, iniciou-se a etapa de regeneração, a qual ocorreu na mesma temperatura da etapa de remoção e com monitoramento do $\mathrm{pH}$. O ar foi borbulhado na solução por um período de $40 \mathrm{~min}$, com pressão constante de 1 bar e vazão de $160 \mathrm{~mL} / \mathrm{min}$. As concentrações de $\mathrm{Fe}^{3+}, \mathrm{Fe}^{2+}$ e ferro total foram analisadas antes e durante a reação, para avaliar a conversão de Fe(II)EDTA em Fe(III)EDTA e a degradação da solução de Fe/EDTA.

\subsection{Determinação das concentrações de ferro total, Fe(II)EDTA e Fe(III)EDTA}

As concentrações dos íons ferros e ferro total foram determinadas com base no método da Fenantrolina (He et al., 2017). Este método baseia-se no fato de que o íon ferroso $\left(\mathrm{Fe}^{2+}\right)$ forma com a orto-fenantrolina, um complexo de cor laranja-avermelhado estável, com absorbância em 510 nm (APHA-AWWA, 1998). A concentração de $\mathrm{Fe}^{3+}$ foi calculada através da diferença entre a concentração de ferro total e a concentração de íons $\mathrm{Fe}^{2+}$. Assim, a degradação do Fe/EDTA é calculada pela redução de ferro total na solução (SAELLE et al., 2012). Todas as análises das concentrações foram feitas em duplicata.

\section{RESULTADOS E DISCUSSÃO}

Os dados de concentrações de ferro total no decorrer da reação para cada temperatura são apresentados na Figura 2. Nota-se que a concentração total de ferro se mantém relativamente constante no decorrer da reação. A degradação de Fe/EDTA é insignificante (< $1,0 \%$ ) para os primeiros 40 minutos para as temperaturas de $15^{\circ} \mathrm{C}, 25^{\circ} \mathrm{C}, 35^{\circ} \mathrm{C}$ e $45^{\circ} \mathrm{C}$. $\mathrm{Na}$ temperatura de $55^{\circ} \mathrm{C}$, identificou-se uma degradação de, aproximadamente, $4,0 \%$ da solução de Fe/EDTA. A degradação do Fe/EDTA, segundo Gambarela et al., é favorecida por altas concentrações de oxigênio e altas temperaturas do meio.

Figura 2 - Concentração de ferro total no decorrer da reação

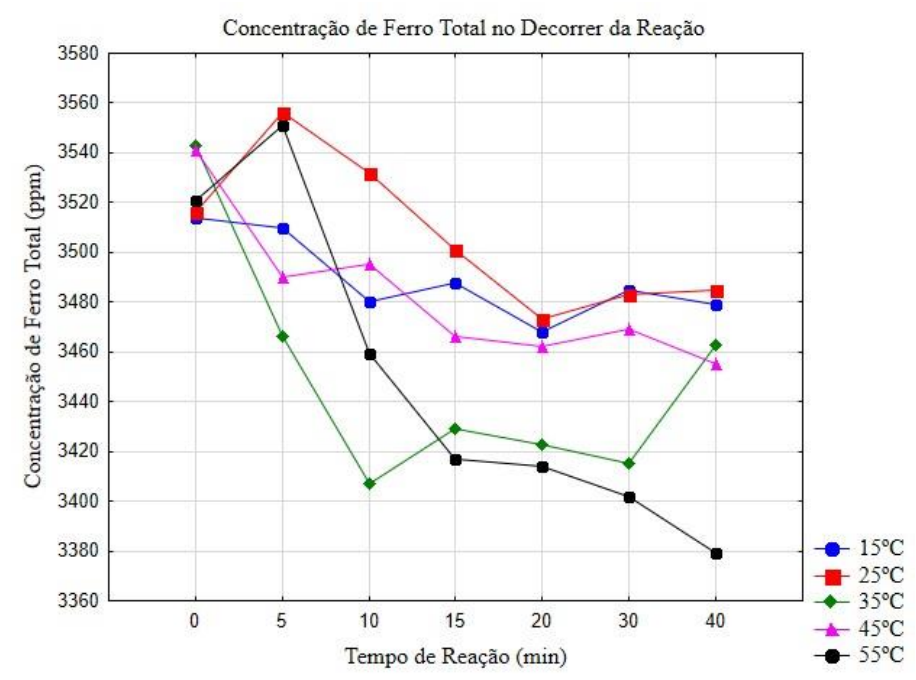




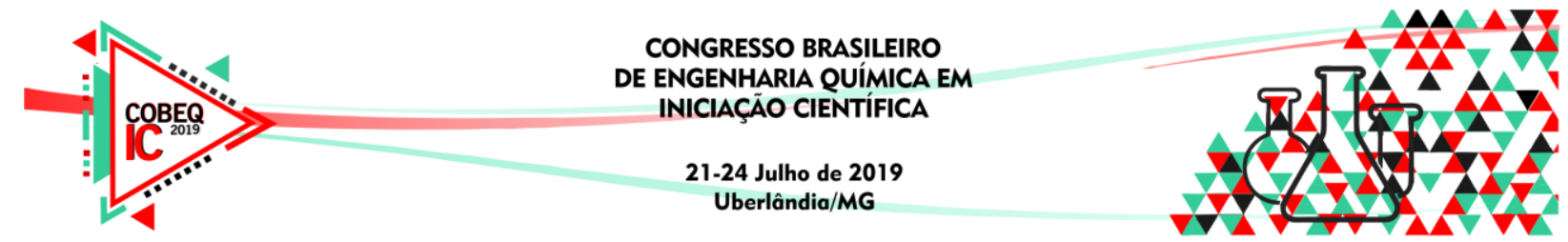

Também foi avaliado a evolução do $\mathrm{pH}$ durante a reação, sendo estes dados apresentados na Figura 3. Observa-se que o pH da solução iniciou em 7,42 $\pm 0,06$, aumentando

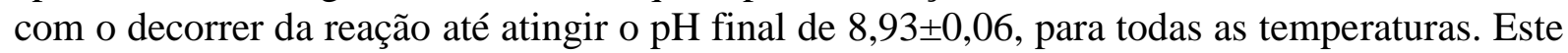
fato pode ser explicado, pois com o decorrer da reação de regeneração, ocorre a redução do $\mathrm{Fe}^{2+}$ para $\mathrm{Fe}^{3+}$, e de acordo com a Equação 4, na qual dois íons $\mathrm{OH}$ - são liberados, acarretando no aumento do $\mathrm{pH}$ da solução.

Figura 3 - pH no decorrer da reação

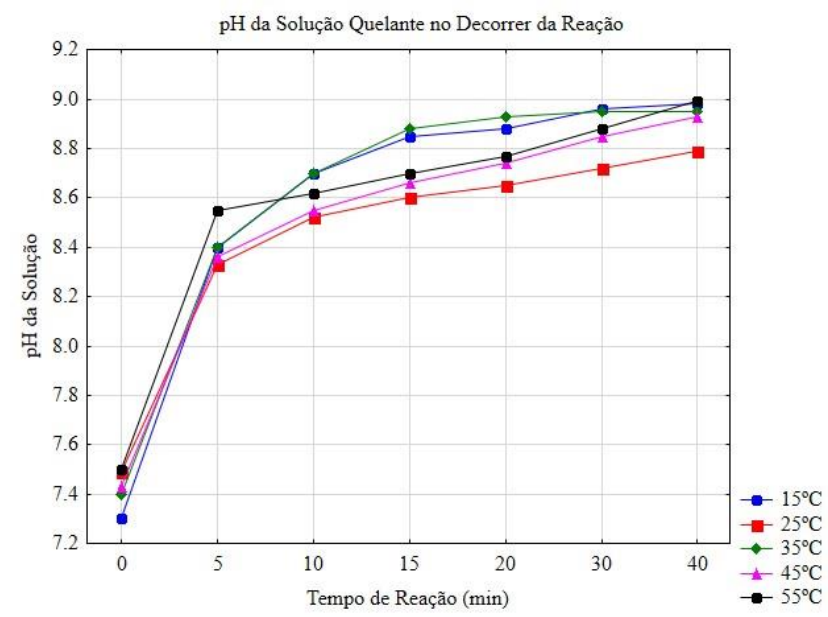

Os dados de concentração de $\mathrm{Fe}^{2+}$ e $\mathrm{Fe}^{3+}$ no decorrer da reação são apresentados na Figura 4 e 5, respectivamente. Como as concentrações inicias de $\mathrm{Fe}^{2+}$ (Figura 4) e as concentrações finais de $\mathrm{Fe}^{3+}$ são aproximadamente constantes, pode-se inferir que houve a mesma quantidade de Fe(II)EDTA oxidada para todas as temperaturas. Quando Fe(II)EDTA é oxidado, há a produção de radicais $\mathrm{OH}^{-}$, o que provoca o aumento do $\mathrm{pH}$ do meio. Como a mesma quantidade de Fe(II)EDTA foi oxidada para todas as temperaturas, houve também a mesma quantidade de hidroxilas produzidas. Desta forma, independente da temperatura em que a reação ocorreu, as soluções finais devem possuir $\mathrm{pH}$ semelhante, como foi observado nos resultados.

Figura 4 - Concentração de $\mathrm{Fe}^{2+}$ no decorrer da reação de regeneração da solução de Fe/EDTA

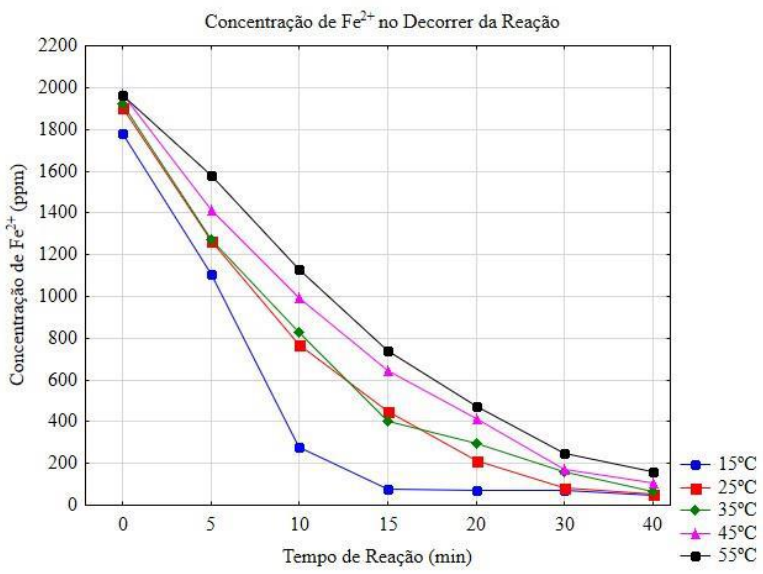




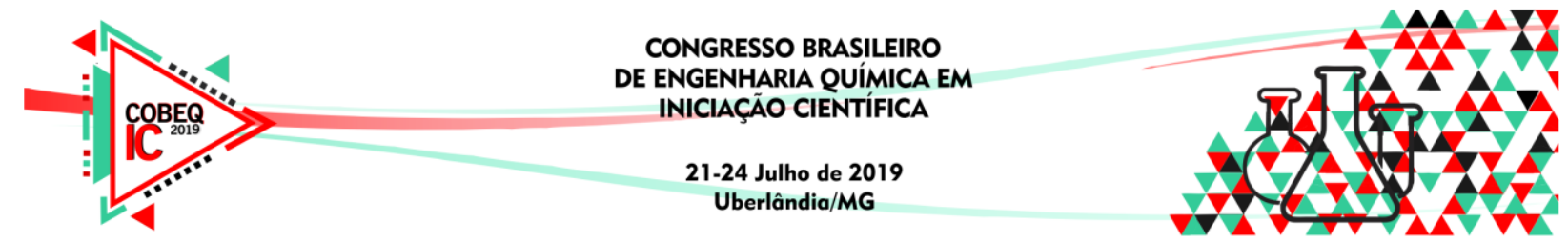

Pode-se notar também que a concentração de $\mathrm{Fe}^{2+}$ cai e, como a quantidade de ferro no sistema se mantém constante, a forma $\mathrm{Fe}^{3+}$ se torna proeminente. Pode-se observar também que com a diminuição da temperatura há o aumento na velocidade da reação e no consumo de $\mathrm{Fe}^{2+} \mathrm{e}$, consequentemente, maior produção de $\mathrm{Fe}^{3+}$ em menor tempo. Segundo Gambardella, a taxa de oxidação de Fe(II)EDTA para Fe(III)EDTA mantem-se praticamente constante para temperaturas acima de $30^{\circ} \mathrm{C}$. Uma hipótese para explicar o aumento da velocidade com a diminuição da temperatura pode se dar pelo aumento da solubilidade do gás oxigênio na solução quelante. De acordo com a medidas experimentais feitas pela YSI Incorporated (Figura 5), a uma pressão de $760 \mathrm{mmHg}$, a quantidade de oxigênio dissolvido em água possui valores maiores para temperaturas mais baixas.

Figura 5 - Variação da solubilidade do oxigênio em água com a temperatura

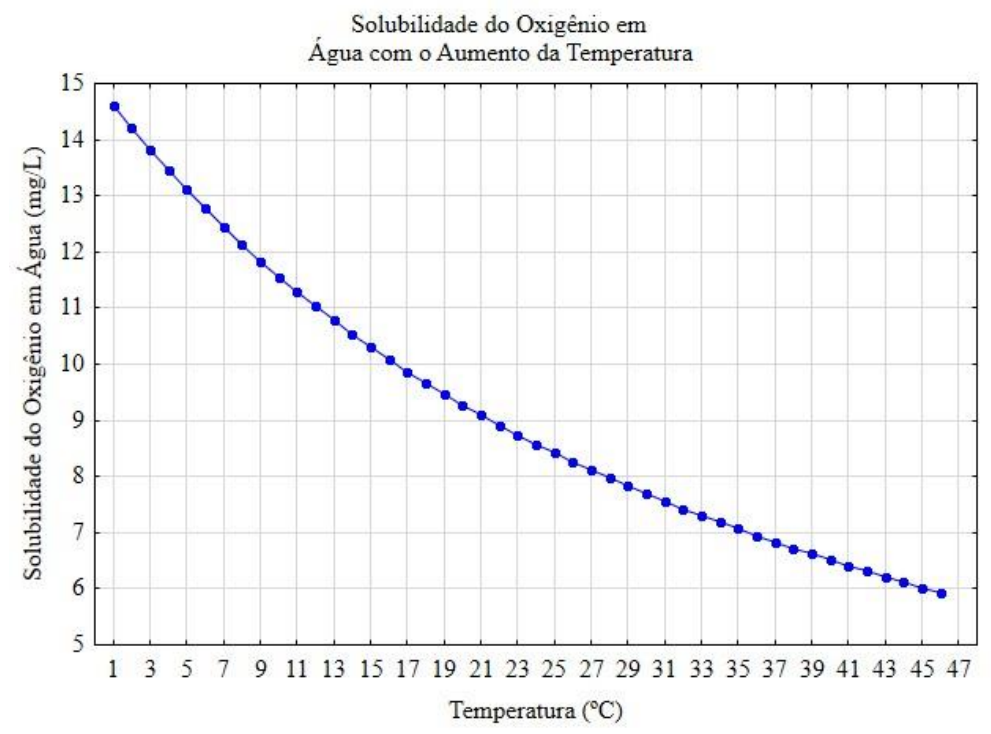

Fonte: YSI Incorporated (adaptado)

No mesmo artigo Gambardella argumenta que a taxa de oxidação é consideravelmente alta para maiores concentrações de oxigênio. Com uma quantidade maior de oxigênio presente no meio, haverá maior taxa de oxidação do Fe(II)EDTA para Fe(III)EDTA, o que deve aumentar o consumo, os mesmos efeitos foram reportados por Zhang et al. e Chi et al.

\section{CONCLUSÃO}

A temperatura influenciou significativamente na velocidade de oxidação do $\mathrm{Fe}(\mathrm{II}) \mathrm{EDTA}$ para $\mathrm{Fe}(\mathrm{III}) \mathrm{EDTA}$. Constatou-se que quanto menor a temperatura do sistema, maior a velocidade de oxidação. A explanação mais provável para este fato é, que com a diminuição da temperatura, há o aumento da solubilidade do gás oxigênio que é o agente oxidante desta reação. Recomenda-se que em trabalhos futuros sejam feitas análises de solubilidade de oxigênio durante a reação visto que o impacto da temperatura não é diretamente nas energias de ativação da reação, mas sim nas concentrações de oxigênio presentes no meio reacional. 


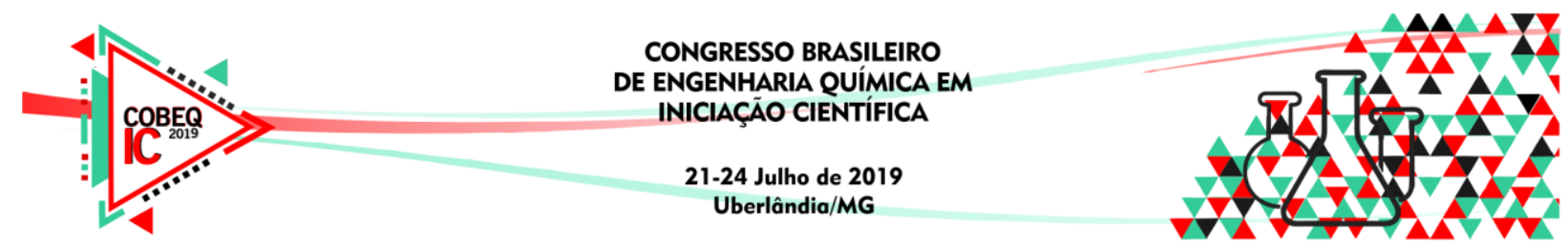

\section{REFERÊNCIAS}

CHI, Y.; XIAO-QIN, W.; DINGQI, W. (2017). Photocatalytic synergistic complex iron denitration regeneration process with iron oxalate. Chinese journal of environmental engineering, v. 12, p 1683-1690.

DESHMUKH,, G. E SHETE, A. (2012). Oxidative Absorption of Hydrogen Sulfide using Iron-chelate Based Process: Chelate Degradation. Analytical \&Bioanalytical Techniques, v. 3, issue3.

FRARE, L. M. Estudos para a Implementação de uma Planta para Remoção de Ácido Sulfídrico de Processos de Produção de Biogás - Tese de Doutorado, PEQ, Universidade Estadual de Maringá, Brasil, 2010.

GAMBARDELLA, F. (2005). NO and O2 absorption in FeII(EDTA) solutions. C. 7, p. 103118.

HE, F.; DENG, X.; CHEN, M. (2017). Evaluation of FE(II)EDTA-NO reduction by zinc powder in wet flue gas denitrification technology with Fe(II)EDTA. Elsevier Fuel 199, p. 523-531.

MAAT, H. T., HOGENDOORNB, J. A., \& VERSTEEG, G. F. (2005). The removal of hydrogen sulfide from gas streams using an aqueous metal sulfate absorbent Part I. The absorption of hydrogen sulfide in metal sulfate solutions. Separation and Purification Technology, v. 43, 183-197.

NADALETTI, W. C., CREMONEZ, P. A., DE SOUZA, S. M., BARICCATTI, R. A., BELLI FILHO, P., \& SECCO, D. (2017). Potential use of landfill biogas in urban bus fleet in the Brazilian states: A review. Renewable and Sustainable Energy Reviews, v. 41, $277-$ 283.

YANG, L., GE, X., WAN, C., YU, F., \& LI, Y. (2014). Progress and perspectives in converting biogas to transportation fuels. Renewable and Sustainable Energy Reviews, 40, 1133-1152.

YSI INCORPORATED (2019). DO - Oxygen Solubility Table. Disponível em: www.ysi.com/File\%20Library/Documents/Technical\%20Notes/DO-Oxygen-SolubilityTable.pdf [Acesso: 14/04/2019].

ZHANG, Q., HU, J., \& LEE, D. (2016 A). Biogas from anaerobic digestion processes: Research updates. Renewable Energy, v. 98, 108-119.

ZHANG, Q.; WANG, S.; ZHANG, G.; ZHU, P. (2016 B). Effects of slurry properties on simultaneous removal of SO2 and NO by ammonia Fe(II)EDTA absorption in sintering plants. Journal of Environmental Management, p 1-7. 